\title{
Successful treatment of ACE inhibitor-induced angioedema with icatibant, a bradykinin B2 receptor antagonist
}

\author{
icatibant may \\ be an effective \\ treatment for \\ severe airway- \\ threatening $A C E$ \\ inhibitor-induced \\ angioedema
}

MBBS(Hons)

Michael O'Sullivan MBBS, FRACP, FRCPA 1,2

Adrian Goudie

MBBS, FACEM, DDU

Timothy Marr MBBS(Hons) ${ }^{3,4}$

Anna Brusch BMBCh, FRACP, FRCPA

1 Fremantle Hospital Perth, WA

2 Princess Margaret Hospital, Perth, WA

3 Sir Charles Gairdner Hospital, Perth, WA.

4 University of Western Australia Perth, WA.

Phillippa.Pucar@ health.wa.gov.au

doi: 10.5694/mjal4.01015

\section{Clinical record}

A 65-year-old woman of European ancestry presented to the emergency department (ED) of our tertiary hospital at 09:30 with isolated tongue swelling without rash, gastrointestinal symptoms or wheeze, which had progressed over the previous 4 hours and was non-responsive to adrenaline administered by her general practitioner at 08:15. She recalled a single episode of mild self-limiting tongue swelling several months earlier and had no family history of angioedema. The patient had well controlled hypertension managed with perindopril ( $5 \mathrm{mg}$ daily) for the past 10 years. Other comorbidities included gastroesophageal reflux disease, hypercholesterolaemia and osteopaenia. She was an active smoker. Other medications included atorvastatin, calcium carbonate, dothiepin, esomeprazole and oestradiol valerate, and she denied over-the-counter medication use.

Despite treatment with intravenous dexamethasone ( $6 \mathrm{mg}$ ), intravenous glycopyrrolate $(0.2 \mathrm{mg})$ and intramuscular promethazine $(25 \mathrm{mg})$, the patient's condition continued to deteriorate. Fibreoptic nasoendoscopy (FNE) revealed epiglottic and left arytenoid oedema. Her treating team planned awake fibreoptic intubation as a priority one case, with a surgical team on standby for emergency tracheostomy.

Icatibant, a competitive bradykinin B2 receptor (B2R)

A CE inhibitors are commonly used in the management of hypertension, and of cardiac and renal disease. Angioedema occurs in $0.1 \%-0.68 \%$ of patients, ${ }_{1}^{1}$ more commonly within 90 days of the introduction of ACE inhibitors, although it can occur later. ${ }^{2}$

After cessation of medication, patients can develop recurrent angioedema, typically within the first month but sometimes several months later. ${ }^{3}$ ACE inhibitors account for $30 \%$ of ED presentations with angioedema, and although most are discharged directly from ED, 11\% require ICU management ${ }^{4}$ for an average of 2.9 days at considerable cost. ${ }^{5}$

ACE inhibitor-induced angioedema is mediated by bradykinin, and empiric therapy with adrenaline, steroids and antihistamines targeted at histaminergic responses is largely ineffective. ${ }^{1}$ Bradykinins bind B2Rs and induce potent vasodilation. ACE (also known as kininase II) promotes bradykinin degradation, so ACE inhibition prolongs the half-life of bradykinin. ${ }^{6}$

Dipeptidyl peptidase-4 (DPP-IV) is a secondary enzyme in bradykinin breakdown. ${ }^{6}$ Concomitant use of ACE inhibitors and DPP-IV inhibitors such as vildagliptin, used to treat type 2 diabetes, or DPP-IV modulation in the context of immunosuppression after transplant, puts patients at increased risk of angioedema.,8 The use of other medications that can cause urticaria and angioedema, such as aspirin or non-steroidal anti-inflammatory drugs, is antagonist, was administered subcutaneously at a dose of $30 \mathrm{mg}$ at 10:30, without development of a local injectionsite reaction. On repeat FNE 10 minutes later, there was a significant improvement in her condition. Intubation was ultimately avoided and she was observed in the intensive care unit (ICU) for 2 hours before being transferred to a highdependency ward area overnight. Complete resolution of symptoms was seen within 5 hours, and she was discharged the next morning after a normal FNE.

Perindopril was implicated in the patient's angioedema. It was discontinued and replaced with amlodipine ( $5 \mathrm{mg}$ daily) and hydrochlorothiazide ( $12.5 \mathrm{mg}$ daily). At follow-up 9 months later, she reported no further episodes of angioedema. Despite thorough investigation, no other cause aside from angiotensin-converting enzyme (ACE) inhibitor-induced angioedema was identified. An infective focus was excluded, with urinalysis, chest $x$-ray and white cell count yielding normal results. Hereditary angioedema and acquired $\mathrm{Cl}$ esterase inhibitor deficiency were excluded by a normal C4 concentration of $0.4 \mathrm{~g} / \mathrm{L}$ (reference interval, $0.16-0.52 \mathrm{~g} / \mathrm{L}$ ) at presentation. Quantitative and functional $\mathrm{Cl}$ inhibitor studies at a subsequent follow-up visit did not find reduced values.

associated with increased risk of angioedema among patients taking ACE inhibitors. ${ }^{6}$

Various epidemiological factors are associated with increased incidence of angioedema with ACE inhibitor use. Patients of African descent have a threefold higher risk compared with those of European descent, ${ }^{8}$ while women have a $50 \%$ higher risk than men. ${ }^{2}$ Patients aged over 65 years, smokers and those subject to trauma, particularly involving the airway, are also at increased risk. ${ }^{6} \mathrm{ACE}$ inhibitor use in heart failure is associated with higher risk compared with its use in hypertension and coronary artery disease, probably due to higher baseline levels of bradykinin. ${ }^{9}$ Our patient, apart from being a female smoker, did not have any additional risk factors. Factors predisposing to ACE inhibitor-induced angioedema are summarised in the Box. ${ }^{6-8}$

Icatibant, a synthetic decapeptide competitive inhibitor of $\mathrm{B} 2 \mathrm{R}$, is a treatment for hereditary angioedema listed on the Pharmaceutical Benefits Scheme. It is administered as a single $30 \mathrm{mg}$ subcutaneous injection at a cost of about $\$ 2500$ per dose.

Given the bradykinin-driven mechanism of ACE inhibitor-induced angioedema, icatibant offers an appealing therapeutic option.

There are no randomised controlled trials examining the use of icatibant in ACE-inhibitor induced angioedema. However, a small case series compared eight patients 
Risk factors for the development of angiotensinconverting enzyme (ACE) inhibitor-induced angioedema

Patient-related factors

- African descent

- Female

- Age $>65$ years

- Smoking

Disease-related factors

- Trauma, especially airway intubation

- Heart failure

Drug-related factors

- Non-steroidal anti-inflammatories and aspirin

- Dipeptidyl peptidase-4 inhibitors, such as sitagliptin and vildagliptin

- Immunosuppressants

- ACE inhibitor-induced cough

Genetic factors

- XPNPEP2 gene polymorphisms (APP gene)

with ACE inhibitor-induced upper-airway angioedema treated with icatibant with 47 historical controls treated with steroids and antihistamines..$^{10}$ Patients receiving icatibant avoided intubation and improved in a mean of 51 minutes, with complete relief at 4.4 hours, compared with 33 hours in the historical controls. ${ }^{10}$

To our knowledge, our case is the first documenting a positive response to icatibant in a patient with an imminently threatened airway. Icatibant is expensive, but the incidence of ACE inhibitor-induced angioedema requiring intubation is fortunately low. Review of local data from Fremantle Hospital, a 450-bed tertiary acute care hospital with 58000 ED presentations annually, identified
Lessons from practice

- Angiotensin-converting enzyme (ACE) inhibitor-induced angioedema is mediated by bradykinin.

- Empiric treatment with steroids, antihistamines and adrenaline is largely ineffective. Angioedema can occur at any time after ACE-inhibitor therapy is initiated.

- Icatibant, a competitive inhibitor of the bradykinin B2 receptor, may be effective in selected patients with airway-threatening angioedema.

120 presentations with angioedema from 2011 to 2013. Of these presentations, seven had severe airway-threatening ACE inhibitor-induced angioedema requiring critical care admission for 1-3 days at an estimated cost of $\$ 5000$ per day. Two patients were intubated at considerable cost in terms of theatre time, equipment and involvement of multiple teams (Dr Yusuf Nagree, Emergency Physician and Academic, Fremantle Hospital, personal communication). Our patient was admitted to the ICU for only 2 hours for observation after receiving icatibant, and avoided intubation.

We propose that icatibant may be an effective treatment for severe airway-threatening ACE inhibitor-induced angioedema, a condition that can be associated with significant morbidity and is typically refractory to other therapies. However, further randomised controlled trial data are needed to confirm efficacy, and evaluation of cost-effectiveness is required before consideration of its use in routine clinical practice.

Competing interests: No relevant disclosures.

References are available online at www.mja.com.au.

of the pathophysiology and risk factors. Clin Exp Allergy 2010; 40: 50-61.

7 Brown NJ, Byiers S, Carr D, et al. Dipeptidyl peptidase-IV inhibitor use associated with increased risk of ACE inhibitorassociated angioedema. Hypertension 2009; 54: 516-523.

8 Stojiljkovic L. Renin-angiotensin system inhibitors and angioedema: anaesthetic implications. Curr Opin Anaesthesiol 2012; 25: 356-362.

9 Makani H, Messerli FH, Romero J, et al. Meta-analysis of randomized trials of angioedema as an adverse event of reninangiotensin system inhibitors. Am J Cardiol 2012; 110: 383-391.

10 Bas M, Greve J, Stelter K, et al. Therapeutic efficacy of icatibant in angioedema induced by angiotensin-converting enzyme inhibitors: a case series. Ann Emerg Med 2010; 56 : 278-282.
5 Baram M, Kommuri A, Sellers SA, Cohn JR. ACE inhibitorinduced angioedema. J Allergy Clin Immunol Pract 2013; 1: 442-445.

6 Hoover T, Lippmann M, Grouzmann E, et al. Angiotensin converting enzyme inhibitor induced angio-oedema: a review
4 Banerji A, Clark S, Blanda M, et al. Multicenter study of patients with angiotensin-converting enzyme inhibitor-induced angioedema who present to the emergency department. Ann Allergy Asthma Immunol 2008; 100: 327-332. 